\title{
PET Imaging of Cancer Immunotherapy
}

Immune system activation can be elicited in viral infections, active immunization, or cancer immunotherapy, leading to the final common phenotype of increased glycolytic use by immune cells and subsequent detection by ${ }^{18} \mathrm{~F}-\mathrm{FDG}$ PET. Because ${ }^{18} \mathrm{~F}-\mathrm{FDG}$ is also used in baseline staging PET/CT scans and in tumor response assessment, physicians are faced with a unique challenge when evaluating tumor response in patients receiving cancer immunotherapy. The burgeoning field of cancer immunotherapy and the paucity of PET probes that can reliably differentiate activated immune cells from metabolically active cancer cells underscore the pressing need to identify and develop additional molecular imaging strategies. In an effort to address this concern, investigators have taken several molecular imaging approaches for cancer immunotherapy. Direct ex vivo labeling of $\mathrm{T}$ lymphocytes with radioactive probes before reinfusion represents the earliest attempts but has proven to be clinically limited because of significant PET probe dilution from proliferation of activated immune cells. Another approach is the indirect in vivo labeling of immune cells via PET reporter gene expression and involves the ex vivo genetic engineering of $T$ lymphocytes with a reporter gene, reinfusion into the host, and the subsequent use of a PET probe specific for the reporter gene. The most recent approach involves the direct in vivo labeling of immune cells by targeting endogenous immune cell biochemical pathways that are differentially expressed during activation. In conclusion, these novel PET-based imaging approaches have demonstrated promise toward the goal of in vivo, noninvasive immune monitoring strategies for evaluating cancer immunotherapy.

Key Words: cancer immunotherapy; adoptive cell transfer; molecular imaging; positron emission tomography; T lymphocyte

J Nucl Med 2008; 49:865-868

DOI: 10.2967/jnumed.108.051342

An initial baseline staging PET/CT scan of a 37-y-old man with newly diagnosed right eye choroidal melanoma demonstrated mild ${ }^{18}$ F-FDG uptake in the right globe corresponding to the mass centered on CT and consistent with the known diagnosis. In addition, moderate to intense ${ }^{18} \mathrm{~F}$-FDG PET uptake in multiple lymph nodes bilaterally in the neck and in the left tracheobronchial region of the chest was noted and reported to be consistent with metastatic disease (Fig. 1). Questioning of the patient elicited a recent upper respiratory tract infection

Received Apr. 23, 2008; revision accepted Apr. 24, 2008.

For correspondence or reprints contact either of the following:

Antoni Ribas, Division of Hematology/Oncology; UCLA Medical Center;

11-934 Factor Building; 10833 Le Conte Ave., Los Angeles, CA 90095-1782.

E-mail: aribas@mednet.ucla.edu

Paul C. Tumeh, Division of Hematology/Oncology; UCLA Medical Center;

11-934 Factor Building; 10833 Le Conte Ave., Los Angeles, CA 90095-1782.

E-mail: ptumeh@mednet.ucla.edu

COPYRIGHT @ 2008 by the Society of Nuclear Medicine, Inc. with a productive cough and fever $3 \mathrm{~d}$ before PET/CT staging. A follow-up PET/CT scan demonstrated complete interval resolution of ${ }^{18} \mathrm{~F}-\mathrm{FDG}$-positive cervical and thoracic adenopathy, with no evidence of malignant disease in the head and neck by ${ }^{18}$ F-FDG PET/CT criteria.

Whether a patient experiences an upper respiratory tract infection or has recently received cancer immunotherapy, the clinical challenge of differentiating metabolically active cancer cells from activated immune cells via conventional radiotracer probes remains the same. This clinical case has been used for illustrative purposes and will be considered analogous to cancer immunotherapy, since both demonstrate an activated host immune system.

Adoptive cell therapy has become one of the great success stories in the field of cancer immunotherapy and is considered to be the most effective therapeutic strategy for patients with metastatic melanoma (1). Briefly, adoptive cell therapy is the administration of a patient's own lymphocytes specific to melanoma antigens after a lymphodepleting regimen. In patients with metastatic melanoma refractory to all other treatments, $50 \%$ have demonstrated an objective response according to the Response Evaluation Criteria in Solid Tumors (RECIST), which is based on CT and MRI linear measurements of lesion size (2). Recently, however, the National Cancer Institute has called for the improvement of the RECIST methodology because these anatomic imaging techniques have proved to be less than optimal predictors of therapeutic response (3). In addition, end-stage anatomic changes in tumor size have not elucidated the reasons why certain patients respond to adoptive cell therapy and others do not.

Molecular imaging strategies using PET hold great promise for detecting molecular changes that manifest shortly after therapy and provide functional information about immune cells that may greatly facilitate advancements in the field of cancer immunotherapy. Specifically, PET enables noninvasive, quantitative, and tomographic assays of biologic processes using positron-emitting radioisotope-labeled probes (4). With this in mind, the conventional PET radiotracer that is routinely used to detect malignancies characterized by an increased rate of glycolysis is ${ }^{18} \mathrm{~F}-\mathrm{FDG}$. However, herein lies the limitation with ${ }^{18} \mathrm{~F}-\mathrm{FDG}$ in the monitoring of transferred immune cells in a cancer setting. The uptake mechanism by which metabolically active cancer cells use this glucose analog has also been demonstrated to be operant in activated immune cells (5-7). Translated to the clinic, in a metastatic melanoma patient receiving adoptively transferred lymphocytes, assessing tumor response via ${ }^{18} \mathrm{~F}$ FDG uptake presents a unique challenge for the clinician 
FIGURE 1. Comparison of ${ }^{18} \mathrm{~F}-\mathrm{FDG}$ PET and ${ }^{18} \mathrm{~F}-\mathrm{FDG}$ PET/CT scans during and after acute upper respiratory tract infection. (A) Patient with ocular melanoma (melanoma lesion not shown) demonstrating moderate to intense ${ }^{18} \mathrm{~F}-\mathrm{FDG}$ PET uptake in multiple lymph nodes bilaterally in neck and chest during infection. (B and $\mathrm{C}$ ) Fused axial PET/CT slices of neck and chest during infection. (D) Follow-up ${ }^{18}$ F-FDG PET scan 2 mo later demonstrating complete interval resolution of ${ }^{18} \mathrm{~F}-\mathrm{FDG}$-positive cervical and thoracic adenopathy. ( $E$ and F) Fused axial PET/CT slices of neck and chest

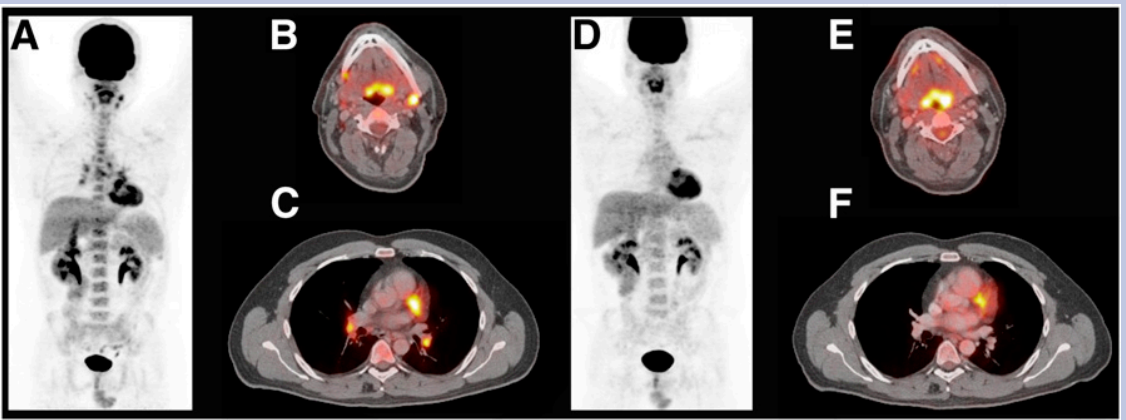
after infection.

because melanoma cells and activated immune cells undergo significant increases in glucose use and a high-intensity PET signal.

To address this limitation, investigatory efforts are currently focused on developing novel PET strategies that can reveal and quantify characteristics unique to immune cells, including immune cell regulatory dynamics, homeostatic expansion, lymphocyte trafficking, and transferred cell persistence. In addition, because adoptive cell therapy is a highly personalized treatment requiring unique reagents for each patient, probes that can accurately provide information on transferred cells may greatly facilitate therapeutic decisions by elucidating how the immune system interacts with cancer. Therefore, measuring these parameters may provide for noninvasive surrogate biomarkers of clinical endpoints, further emphasizing the need to identify such probes.
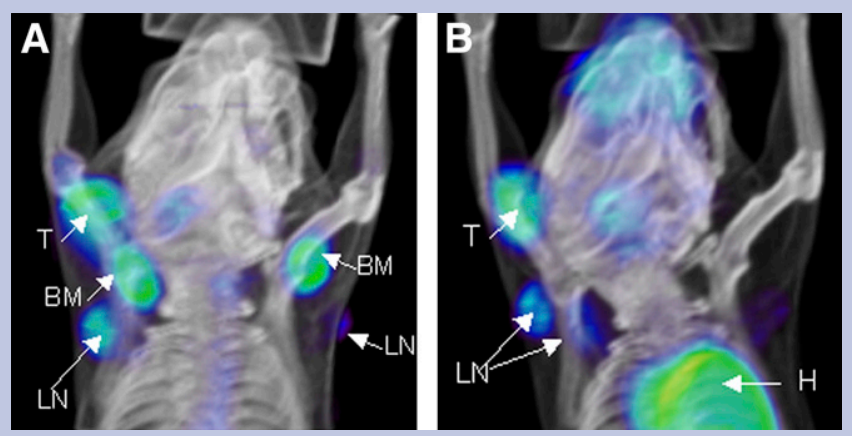

FIGURE 2. Bone marrow chimeric mice were generated by engraftment of hematopoietic stem and progenitor cells transduced with trifusion reporter gene encoding synthetic Renilla luciferase, enhanced green fluorescent protein, and HSV-tk. Mice bearing rhabdomyosarcomas were challenged with Moloney murine sarcoma and leukemia virus complex, and induced immune response was monitored via ${ }^{18} \mathrm{~F}-\mathrm{FDG}$ and ${ }^{18} \mathrm{~F}$ FHBG PET. (A) ${ }^{18} \mathrm{~F}-\mathrm{FHBG}$ retention was detected in tumor and draining lymph nodes between days 8 and 14 after tumor challenge. (B) Using ${ }^{18} \mathrm{~F}-\mathrm{FDG}$, activated immune cells were observed at tumor draining lymph nodes between days 13 and 15. $\mathrm{BM}=$ bone marrow; $\mathrm{H}=$ heart; $\mathrm{LN}=$ lymph node; $\mathrm{T}=$ tumor.
Several immune cell PET strategies have been developed and can be categorized into direct and indirect imaging protocols: direct ex vivo labeling of immune cells, indirect in vivo labeling of immune cells via PET reporter gene expression, and direct in vivo labeling of immune cells via targeting of endogenous intracellular proteins.

Direct ex vivo labeling represents the earliest imaging strategy and was initially used to visualize the biodistribution of reinfused T lymphocytes $(8,9)$. These studies demonstrated that $\mathrm{T}$ lymphocytes incorporated significant amounts of ${ }^{18} \mathrm{~F}$-FDG but released it shortly thereafter because of phosphatase activity. PET could follow their trafficking for 24-36 h, providing for a small window of opportunity to study in vivo lymphocyte trafficking. Significantly, however, this strategy precludes long-term immune cell monitoring because apoptosis or proliferation of these cells in vivo would lead to detrimental levels of probe dilution.

Indirect in vivo labeling via PET reporter gene constructs addresses the probe dilution problem and allows for serial imaging of cell trafficking and homeostatic expansion (Fig. 2) (10-12). Genetic engineering enables cells and their progeny to express a PET reporter gene that codes for a differentially expressed protein leading to enhanced uptake of the radiotracer. Several reviews have been provided for a more in depth discussion of the concepts, challenges, and advantages of reporter gene strategies (12-14). Herpes simplex virus type 1 thymidine kinase (HSV1-tk) and its mutated derivative HSV-sr39tk have experienced the greatest advances in PET reporter gene strategies. Briefly, HSV1-tk converts thymidine to its phosphorylated form in addition to pyrimidine analogs such as acyclovir, ganciclovir, and penciclovir, leading to intracellular trapping (15). Positronemitting pyrimidine analogs, such as $9-\left(4-{ }^{18} \mathrm{~F}-\right.$ fluoro-3[hydroxymethyl]butyl)guanine $\left({ }^{18} \mathrm{~F}-\mathrm{FHBG}\right)$ and $2^{\prime}$-flouro- $2^{\prime}$ deoxy-1-B-D-arabinofuranoysluracil (FIAU), have subsequently demonstrated preferential intracellular trapping by cells transduced with the HSV1-tk PET reporter gene. The use of picomolar amounts of these labeling substrates ensures against cellular toxicity observed after administration of pharmacologic doses of ganciclovir. Therefore, HSV1-tk and HSV-sr39tk serve a dual function in that, at 
low doses, imaging can be performed and, at pharmacologic doses, they serve as a suicide gene, protecting the patient from the potential of malignantly transformed genetically engineered cells or life-threatening autoimmunity.

Several groups have used PET reporter gene strategies in adoptively transferred lymphocytes. Dubey et al. (16) demonstrated that small-animal PET could quantify adoptively transferred $\mathrm{T}$ lymphocytes eliciting an antitumor response in whole animals. $\mathrm{T}$ lymphocytes from animals that had rejected a murine sarcoma were then transduced with HSV-sr39tk, injected into tumor-bearing mice, and imaged by small-animal PET. Localization of the adoptively transferred $\mathrm{T}$ cells in the antigen-positive tumor was detected by sequential imaging of the animals. In another study, Su et al. (17) correlated PET signal intensity with the number of cells in a region of interest. Different numbers of primary $\mathrm{T}$ lymphocytes were retrovirally transduced with the HSV-sr39tk and intratumorally injected followed by ${ }^{18} \mathrm{~F}$-FHBG administration, providing for a proof-of-concept study that reporter genes could quantify transferred cell numbers via PET signal. Koehne et al. (18) demonstrated that Epstein-Barr virus (EBV)-specific T cells transduced with HSV1-tk could preferentially accumulate radiolabeled FIAU. After adoptive transfer, T lymphocytes labeled with ${ }^{124}$ I-FIAU could be tracked by PET in severe combined immunodeficiency mice bearing human tumor xenografts.

In another strategy, investigators are exploring the use of a reporter gene that codes for a cell surface receptor. Kenanova et al. provided data (unpublished) that a modified carcinoembryonic antigen gene could potentially be used as a reporter gene for clinical application. Theoretically, this approach may provide for a more facile method in quantifying PET signal with immune cell number when compared with intracellular enzymatic reporters, because a stoichiometric binding of probe and receptor is required. However, because this method lacks signal amplification, it requires a high number of cell surface receptors to be expressed and may experience challenges in the clinic from low sensitivity.

As reporter gene imaging strategies continue to advance, several key issues must be addressed to ensure successful translation of this strategy into the clinic. The first is sensitivity: lymphocyte trafficking leads to a diffuse distribution throughout the body. PET visualization of these cells must be able to detect relatively small numbers of cells and provide for high resolution at a given anatomic location. The second is specificity: reporter gene proteins should be differentially expressed in immune cells with minimal endogenous expression, providing for an adequate signal-to-noise ratio. The third is toxicity: reporter gene proteins must not induce cell death or altered function at doses required for imaging. The last is immunogenicity: the reporter gene protein must not be immunogenic because that could lead to a host response to the transferred cells.

Most recently, Doubrovin et al. (19) explored the use of a human norepinephrine transporter and used a previously approved clinical-grade radiolabeled probe, metaiodobenzylguanidine. When human norepinephrine transporterpositive EBV-specific T cells were infused, PET enabled imaging over $28 \mathrm{~d}$ of their migration and specific accumulation in EBV-positive tumor xenografts expressing their restricted human leukocyte antigen allele. Ponomarev et al. (20) made a significant step toward a clinical-grade PET reporter gene when they demonstrated that a variant of thymidine kinase, human thymidine kinase 2 , which is normally restricted in expression to the mitochondria, could be expressed in the cytosol of transduced cells via N-terminus truncation and intracellularly trap PET probes.

Although significant progress has been made in the field of PET reporter gene strategies, an insoluble challenge lies in the extensive in vitro genetic manipulation of lymphocytes before reinfusion, potentially altering cell viability and function. This challenge has led investigators to take on another strategy: direct in vivo labeling of endogenous proteins in immune cells. Specifically, PET of metabolic signatures unique to activated immune cells may provide for a clinically significant imaging strategy.

Radu et al. (21) demonstrated a proof-of-concept study that exploited biochemical pathways as an endogenous PET reporter. In experimental autoimmune encephalitis, a murine model of multiple sclerosis, ${ }^{18} \mathrm{~F}$-FDG enabled visualization of spinal cord inflammation through activated lymphocytes. However, whereas ${ }^{18}$ F-FDG PET could monitor response to corticosteroid therapy, the magnitude of uptake did not correlate with disease progression in animals with advanced disease. This initial work in imaging endogenous pathways led the group to subsequently focus on metabolic signatures that may be more unique to immune cell activation than glycolysis.

Although most tissues predominantly use the de novo pathway for DNA synthesis, lymphoid organs extensively use the salvage pathway. A systematic approach was created where in vitro screening of nucleoside analogs for differential retention in proliferating and resting $\mathrm{T}$ lymphocytes was performed. A novel PET probe, 1-(2-deoxy-2flouro-arabinofuranosyl)-cytosine (FAC) was identified as having significant accumulation in proliferating $\mathrm{T}$ cells (22). Subsequent radiochemical synthesis of ${ }^{18}$ F-FAC demonstrated greater specificity for lymphoid organs than PET probes for nucleoside metabolism ( $\left.{ }^{18} \mathrm{~F}-\mathrm{FLT},{ }^{18} \mathrm{~F}-\mathrm{FMAU}\right)$ and glycolysis $\left({ }^{18} \mathrm{~F}-\mathrm{FDG}\right)$. Significantly, ${ }^{18} \mathrm{~F}$-FAC was further shown to preferentially accumulate in tumor-draining lymph nodes of oncoretrovirus-induced tumors in mice when compared with ${ }^{18} \mathrm{~F}$-FDG and ${ }^{18} \mathrm{~F}$-FLT. This work demonstrated that by exploiting metabolic signatures in activated immune cells, direct in vivo labeling served as a promising platform for PET probe development.

PET of cancer immunotherapy has made great progress in visualizing and quantifying lymphocyte homing and trafficking, homeostatic expansion, and transferred cell persistence. PET probes that can image these parameters may greatly facilitate therapeutic decisions in an accurate and 
timely manner by elucidating how the immune system interacts with cancer. Two main strategies are currently being investigated: reporter gene imaging and endogenous gene imaging. Humanized reporters and exploitation of biochemical signatures in activated immune cells are promising additional approaches to address current challenges.

\section{Paul C. Tumeh \\ Caius G. Radu \\ Antoni Ribas \\ UCLA \\ Los Angeles, California}

\section{ACKNOWLEDGMENTS}

This research is supported in part by the Scholars in Molecular Imaging Fellowship and by grant P50 CA086306 from the National Institutes of Health.

\section{REFERENCES}

1. Rosenberg SA, Restifo NP, Yang JC, Morgan RA, Dudley ME. Adoptive cell transfer: a clinical path to effective cancer immunotherapy. Nat Rev Cancer. 2008;8:299-308.

2. Dudley ME, Wunderlich JR, Yang JC, et al. Adoptive cell transfer therapy following non-myeloablative but lymphodepleting chemotherapy for the treatment of patients with refractory metastatic melanoma. J Clin Oncol. 2005; 23:2346-2357.

3. Czernin J, Weber WA, Herschman HR. Molecular imaging in the development of cancer therapeutics. Annu Rev Med. 2006;57:99-118.

4. Czernin J, Phelps ME. Positron emission tomography scanning: current and future applications. Annu Rev Med. 2002;53:89-112.

5. Frauwirth KA, Riley JL, Harris MH, et al. The CD28 signaling pathway regulates glucose metabolism. Immunity. 2002;16:769-777.

6. Frauwirth KA, Thompson CB. Regulation of $\mathrm{T}$ lymphocyte metabolism. J Immunol. 2004;172:4661-4665.

7. Shu CJ, Guo S, Kim YJ, et al. Visualization of a primary anti-tumor immune response by positron emission tomography. Proc Natl Acad Sci USA. 2005; 102:17412-17417.
8. Adonai $\mathrm{N}$, Nguyen $\mathrm{KN}$, Walsh $\mathrm{J}$, et al. Ex vivo cell labeling with ${ }^{64} \mathrm{Cu}-$ pyruvaldehyde-bis(N4-methylthiosemicarbazone) for imaging cell trafficking in mice with positron-emission tomography. Proc Natl Acad Sci USA. 2002; 99:3030-3035.

9. Botti C, Negri DR, Seregni E, et al. Comparison of three different methods for radiolabelling human activated T lymphocytes. Eur J Nucl Med. 1997;24:497504.

10. Herschman HR. Noninvasive imaging of reporter gene expression in living subjects. Adv Cancer Res. 2004;92:29-80.

11. Herschman HR. PET reporter genes for noninvasive imaging of gene therapy, cell tracking and transgenic analysis. Crit Rev Oncol Hematol. 2004;51: 191-204.

12. Massoud TF, Gambhir SS. Molecular imaging in living subjects: seeing fundamental biological processes in a new light. Genes Dev. 2003;17:545-580.

13. Gambhir SS, Barrio JR, Herschman HR, Phelps ME. Assays for noninvasive imaging of reporter gene expression. Nucl Med Biol. 1999;26:481-490.

14. Nair-Gill ED, Shu CJ, Radu CG, Witte ON. Non-invasive imaging of adaptive immunity using positron emission tomography. Immunol Rev. 2008;221: 214-228.

15. Tjuvajev JG, Avril N, Oku T, et al. Imaging herpes virus thymidine kinase gene transfer and expression by positron emission tomography. Cancer Res. 1998;58:4333-4341.

16. Dubey $\mathrm{P}, \mathrm{Su} \mathrm{H}$, Adonai $\mathrm{N}$, et al. Quantitative imaging of the $\mathrm{T}$ cell antitumor response by positron-emission tomography. Proc Natl Acad Sci USA. 2003; 100:1232-1237.

17. Su H, Forbes A, Gambhir SS, Braun J. Quantitation of cell number by a positron emission tomography reporter gene strategy. Mol Imaging Biol. 2004;6:139-148.

18. Koehne G, Doubrovin M, Doubrovina E, et al. Serial in vivo imaging of the targeted migration of human HSV-TK-transduced antigen-specific lymphocytes. Nat Biotechnol. 2003;21:405-413.

19. Doubrovin MM, Doubrovina ES, Zanzonico P, Sadelain M, Larson SM, O'Reilly RJ. In vivo imaging and quantitation of adoptively transferred human antigenspecific T cells transduced to express a human norepinephrine transporter gene. Cancer Res. 2007;67:11959-11969.

20. Ponomarev V, Doubrovin M, Shavrin A, et al. A human-derived reporter gene for noninvasive imaging in humans: mitochondrial thymidine kinase type 2 . $J$ Nucl Med. 2007;48:819-826.

21. Radu CG, Shu CJ, Shelly SM, Phelps ME, Witte ON. Positron emission tomography with computed tomography imaging of neuroinflammation in experimental autoimmune encephalomyelitis. Proc Natl Acad Sci USA. 2007;104: 1937-1942.

22. Radu CG, Shu CJ, Nair-Gill ED, et al. Molecular imaging of lymphoid organs and immune activation using positron emission tomography with a new ${ }^{18} \mathrm{~F}$-labeled 2 -deoxycytidine analog. Nat Med. In press. 